\title{
Studying molecular profiles above the Cherenkov Telescope Array sites
}

\author{
Pere Munar-Adrover ${ }^{1 *}$ and Markus Gaug ${ }^{1, * *}$ for the CTA Consortium ${ }^{* * *}$ \\ ${ }^{1}$ Unitat de Fisica de les Radiacions, Departament de Fisica and CERES-IEEC, Universitat Autonoma de Barcelona, 08193 Bellaterra, \\ Spain
}

\begin{abstract}
The Cherenkov Telescope Array (CTA) will bring a whole new insight to the gamma-ray Universe. In order to fulfill its performance requirements, we need to understand and correct the atmospheric effects that influence the acquired instrument data. One such systematic effect is due to the varying molecular density profile with time. We have studied such profiles for both CTA sites using publicly available historical data assimilation archives. Our study reveals that we can distinguish at least three differentiated seasonal periods at the northern site and at least two at the southern site, that allow to model the molecular part of the atmosphere using average profiles, as done with current Cherenkov telescope projects. Seasonal transitions are smoother at the southern site than at the northern one. Moreover, the latter shows a greater amplitude in density variations at an altitude of $15 \mathrm{~km}$. We also explored deviations of the molecular profiles with respect to their mean values using a 5-years data set and concluded that they are always found within specifications.
\end{abstract}

\section{Introduction}

Imaging Atmospheric Cherenkov Telescopes (IATCs) observe very-high energy gamma rays indirectly from the ground through the Cherenkov light emitted when the radiation interacts with the atmosphere. The characteristics of the Cherenkov light-cone produced depend on the state of the atmosphere, particularly on the molecular profile [1]. Later on, the transmission of Cherenkov light through the atmosphere changes with its molecular and aerosol content. Thus, this observational technique is prone to a series of systematic effects due to the uncertainties inherent to the local climate and weather conditions. In particular, changes of the local density profile [1-3] and the aerosol content [4-7] are its main contributions.

Up to now, IATC collaborations have modeled the atmosphere using one average density profile, tailored to each particular site. The next generation of IACTs, embodied in the Cherenkov Telescope Array (CTA) [8], with more than one hundred IATCs distributed over two sites, one in the Northern Hemisphere at the Observatorio del Roque de los Muchachos, on the island of La Palma, Spain, and one in the Southern Hemisphere located at the Atacama desert close to Paranal, in northern Chile, has much more stringent requirements on allowed systematic uncertainties than previous installations, for instance those affecting assumed absolute energy scale shall be brought from currently $15-20 \% \quad[2,4,5]$ down to a level of the less than $10 \%$, across a wider energy range.

Here, we study the molecular profiles from data provided by modern data assimilation systems.

\footnotetext{
*e-mail: pere.munar@uab.cat

**e-mail: markus.gaug@uab.cat

***www.cta-observatory.org
}

\section{The global data assimilation archives}

There are many meteorological data assimilation systems (DAS) available, each of them with different characteristics in terms of availability, spatial and temporal resolution, physical parameters and height levels, among others. In order to approach the problem of characterizing the atmosphere above the two CTA sites, we wanted to take into account those, which are easily accessible for our locations of interest, updated with good spatial and temporal resolution and with all the physical parameters available that we need to compute the density profiles. For this reason, after reviewing most of the available DAS, we chose to use two: the National Centers for Environmental Prediction (NCEP) Final Analysis obtained from the Global Data Assimilation System (GDAS) ${ }^{1}$ and the European Centre for Medium-Range Weather Forecasts (ECMWF) ERAInterim reanalysis ${ }^{2}$. The main characteristics of these two DAS are summarized in Table 1.

\subsection{NCEP Final Analysis}

This DAS model consists of a continuous analysis of the data from the Global Data Assimilation System (GDAS from now on), collected by the Global Telecommunications System (GTS) and other sources. The dataset is structured in a grid of $1^{\circ} \times 1^{\circ}$ resolution, with data provided every $6 \mathrm{~h}$ at 26 pressure levels, from surface at $1000 \mathrm{hPa}$ up to $10 \mathrm{hPa}$. For the purpose of our study, among the many available physical parameters, we selected geopotential height, temperature, relative humidity and $u$ - and $v$-components of the wind.

\footnotetext{
${ }^{1}$ http://www.emc.ncep.noaa.gov/index.php?branch=GFS

${ }^{2}$ https://www.ecmwf.int/
} 
Table 1: Data assimilation systems overview

\begin{tabular}{lll}
\hline & ERA-Interim & GDAS \\
\hline Availability & $1979-$ present & 2006 - present \\
Grid spacing & $0.75^{\circ}$ & $1.0^{\circ}$ \\
Temporal resolution & $6 \mathrm{~h}$ & $6 \mathrm{~h}$ \\
Selected dataset time span & $2012 / 01-2016 / 12$ & $2012 / 01-2016 / 12$ \\
Closest grid point North & $28.5^{\circ} \mathrm{N} 18.0^{\circ} \mathrm{W}$ & $29.0^{\circ} \mathrm{N} 18.0^{\circ} \mathrm{W}$ \\
Closest grid point South & $24.75^{\circ} \mathrm{S} 70.5^{\circ} \mathrm{W}$ & $25.0^{\circ} \mathrm{S} 70.0^{\circ} \mathrm{W}$ \\
Pressure levels & 37 & 26 \\
\hline
\end{tabular}

The data can be downloaded for a specific grid point via a python script and are stored in grib2 format, readable also with a python script that we developed. For our work, and due to the limited spatial resolution, we selected the closest grid-point to the locations of both CTA sites, namely $29.0^{\circ} \mathrm{N} 18.0^{\circ} \mathrm{W}$ for the northern site and $25.0^{\circ} \mathrm{S}$ $70.0^{\circ} \mathrm{W}$ for the southern site.

\subsection{ECMWF ERA-Interim}

The ECMWF offers the re-analysis (ERA-Interim) of data based on the 2006 release of the Integrated Forecasting Systen (IFS). It consists of a dataset that spans from 1979 to present, provided with 2 months delay with respect to the present date.

The data are structured in 37 pressure levels, from surface level up to $1 \mathrm{hPa}$. Among all available parameters, for the purposes of this work we selected the geopotential, temperature, $u$ - and $v$-components of the wind and relative humidity. The spatial resolution of the dataset is $0.75^{\circ}$ and its temporal resolution is $6 \mathrm{~h}$.

Data can be downloaded for a specific grid point through a python script. We selected the closest gridpoints available, namely $28.5^{\circ} \mathrm{N} 18.0^{\circ} \mathrm{W}$ for the Northern Hemisphere and $24.75^{\circ} \mathrm{S} 70.5^{\circ} \mathrm{W}$ for the Southern Hemisphere site.

\section{Results}

We downloaded data from 2012 to 2016 (5 complete years) for both CTA sites and for each one of the two selected DAS. Our scope was to compare these datasets and study the evolution of the molecular profile data at each site along these five years.

In all cases, we computed the density for each pressure level and for each moment in time. We then multiplied the density by the standard atmosphere density $\left(N_{\mathrm{S}}\right)$ and an exponential of the height divided by the standard height $\left(H_{\mathrm{S}}\right)$, in order to obtain plots with good visibility of the relative differences. After that, we computed the average density, standard deviation and peak-to-peak extreme values for each pressure level. We also compared the obtained results to those used in recent simulations [9], labelled PROD3 in the figures.

\subsection{Density at 15 km}

First we looked at the density as a function of time for a fixed value of height (or fixed pressure level). We chose to look at $15 \mathrm{~km}$ height, at the onset of the lower stratosphere, where deviations are expected to be largest. The comparison between both sites (see Figure 1) shows that the annual variations are smoother at the southern site and that the amplitude is larger at the northern site.

With this in mind, we can define different seasonal periods for each site, within which the density profile can be averaged (see Figure 1). We define three of these seasonal periods for the northern site (that we call winter, summer and intermediate) and two for the southern site (that we call winter and summer).

The El Niño and La Niña phenomena are to be taken into account for the southern site, since they strongly affect the climate at this particular region of the planet. Taking a look into the historical data, we observe that El Niño was active during 2014-2016 and it was one of the strongest El Niño events ever registered. Despite that, Figure 1 shows no visible deviation for this particular period, with respect to other years. This might indicate that El Niño will not affect much the state of the molecular profile of the atmosphere at the southern site.

\subsection{Density over altitude}

We compared the two data-sets by looking at the density over altitude. In Figure 2, we plot the mean density and its standard deviation as a function of altitude, for each DAS seasonal period. The behaviour is very similar for ECMWF and GDAS, and the relative differences are small between them. However, they are bigger in the southern site than in the northern one, probably due to the fact that the southern site has less coverage of close-by radio-sonde data compared to the northern one. This fact is reflected in Figure 4 where relative differences between the two different DAS used are displayed as a function of altitude. In both cases the differences are centered around zero value, but in the south the dispersion is higher than in the north. It is also visible the fact that these differences are more important at altitudes above $\sim 16 \mathrm{~km}$ in the north and $\sim 12 \mathrm{~km}$ in the south, probably due to the fact that GDAS has less information at the tropopause level. Nonetheless, looking at Figure 2, the different seasonal periods can be clearly distinguished above about $12 \mathrm{~km}$ altitude. The bottom parts of Figure 2 show the behaviour of the density 


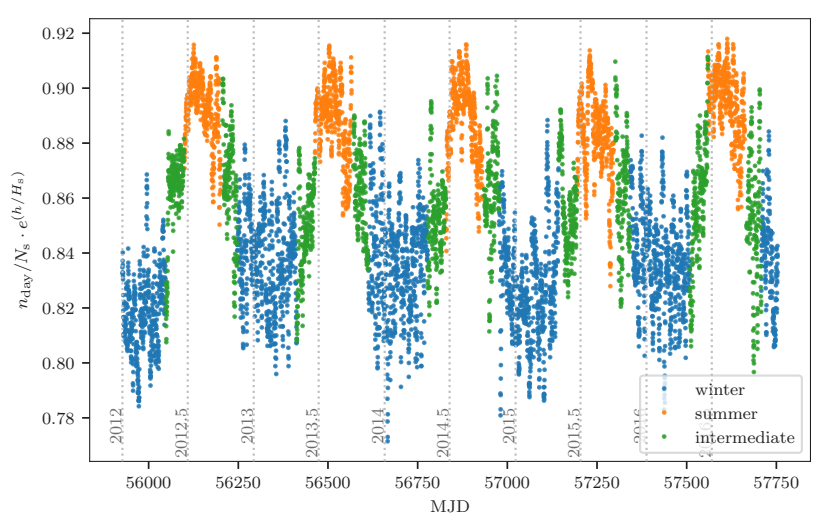

(a) ECMWF-ERA Interim North

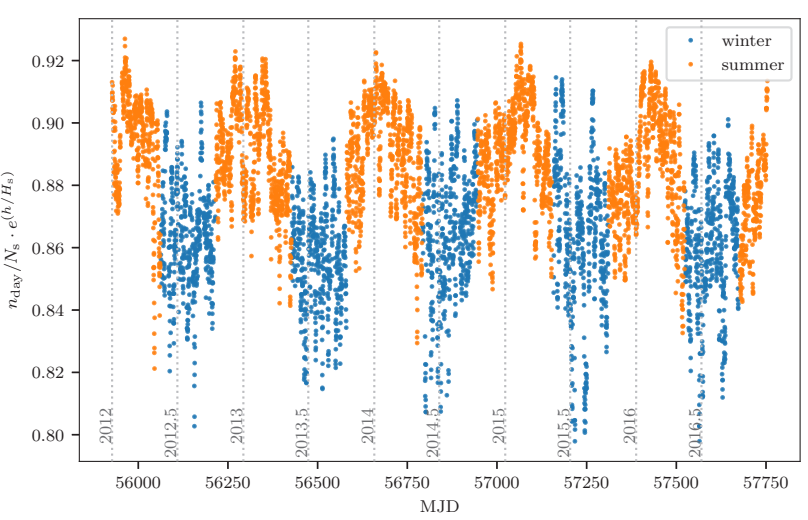

(b) GDAS South

Figure 1: Scaled density at $15 \mathrm{~km}$ altitude. Different colors represent the various seasonal periods that we defined according to the observed behavior in these plots.

standard deviation divided by its mean, for each seasonal periods. That relative deviation lies always below $2 \%$, hence fulfilling the CTA requirements [10].

Moreover, we clearly see that there is a significant difference with respect to the PROD3 simulation profile, reaching up to $12 \%$ on average for one altitude bin (see Figure 3) at the northern CTA site. We find our respective summer DAS profiles closer to the profiles used for the PROD3 simulations than the winter ones. Normally, telescopes take more data during winter, when the nights are longer. However, the differences are small enough not to alter the sensitivity comparisons of [9]. Figure 3 also justifies the selection of our suggested three separated seasonal periods for the north, instead of using only one average model. In the case of the southern site, these differences are more subtle, but still visible by eye.

Finally we used these data to produce CORSIKA input card files that will be used for future simulations of air showers.

\section{Conclusions}

We investigated the long term variation of the molecular density profiles above the two selected sites for the CTA. In this work, we observe that in the southern hemisphere the transitions between seasons are smoother than in the northern hemisphere and that the amplitude in density variations is larger in the latter. Moreover, we identify two distinct seasonal periods at the southern and three at the northern site.

Comparing the density profiles, we can distinguish different behaviours depending on the seasonal period and that the profiles used for the PROD3 simulations so far resemble a summer profile (for the northern site) and a winter profile (for the southern site). The differences between our profiles and those used for the simulations can be as larger as $9 \%$ on average for a selected altitude bin.
The comparison between GDAS and ECMWF reveals that both datasets are very similar. ECMWF offers more pressure levels and is easier to download, but aside of that, we can conclude that for the purposes of this study they are almost equivalent.

\section{References}

[1] K. Bernlöhr, Astroparticle Physics 12, 255 (2000), astro-ph/9908093

[2] M.K. Daniel, Application of radiosonde data to VERITAS simulations, in Proc. 30th International Cosmic Ray Conference, Mérida (2008), Vol. 3, pp. 1329_ 1332

[3] M. Gaug, CTA Atmospheric Calibration, in European Physical Journal Web of Conferences (2017), Vol. 144, p. 01003

[4] F. Aharonian et al., Astronomy \& Astrophysics 457, 899 (2006)

[5] J. Aleksić et al., Astroparticle Physics 72, 76 (2016), arXiv:1409.5594

[6] S.J. Nolan, G. Pühlhofer, C.B. Rulten, Astroparticle Physics 34, 304 (2010), arXiv:1009.0517

[7] D. Garrido, M. Gaug, M. Doro, L. Font, A. LópezOramas, A. Moralejo, Atmospheric Aerosols at the MAGIC Site, in Proceedings of the $33^{\text {rd }}$ ICRC, Rio de Janeiro (2013), p. 0465, arXiv:1308.0473

[8] B.S. Acharya et al., Astroparticle Physics 43, 3 (2013)

[9] T. Hassan, L. Arrabito, K. Bernlöhr, J. Bregeon, J. Cortina, P. Cumani, F. Di Pierro, D. FalcetaGoncalves, R.G. Lang, J. Hinton et al., Astroparticle Physics 93, 76 (2017), arXiv: 1705.01790

[10] M. Vrastil, Overview of Atmospheric Simulation Efforts in CTA, in AtmoHEAD 2016, Olomouc, Czech Republic; EPJ Web of Conferences, Volume 144, id.01014 (2017), Vol. 144 


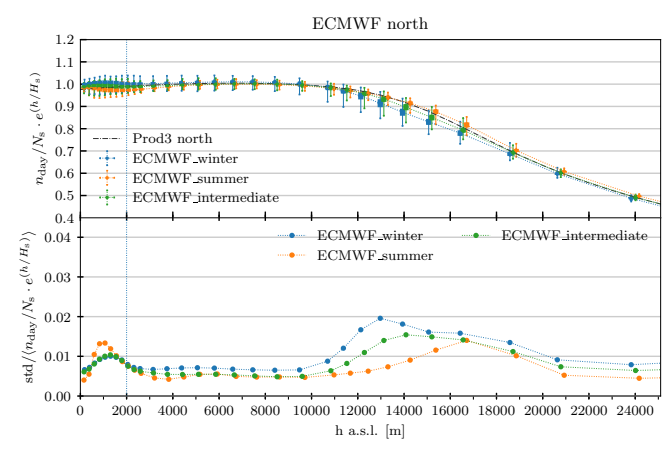

(a) North

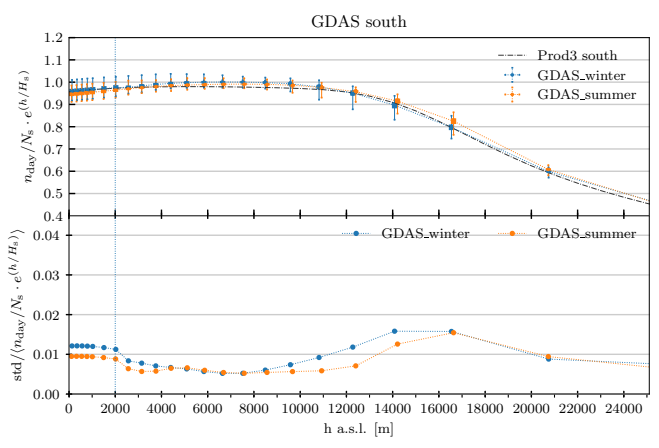

(b) South

Figure 2: Scaled density as a function of altitude. The thick error bars represent the standard deviation of the scaled density and the thin error bars the peak-to-peak values at each altitude bin. The dotted vertical line marks the $2000 \mathrm{~m}$ line, at which the observatories are placed.

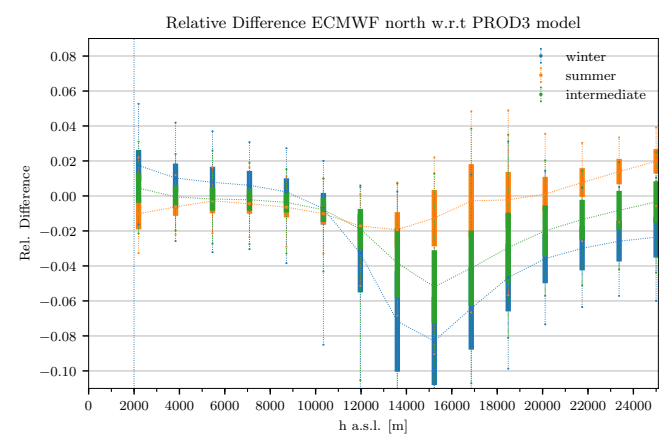

(a) North

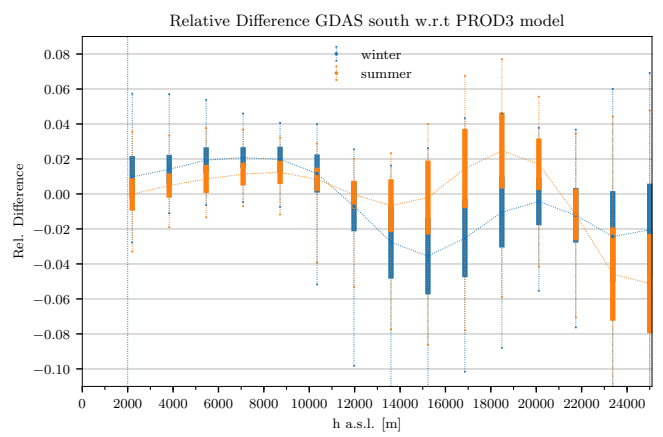

(b) South

Figure 3: Relative difference between the computed density from ECMWF-ERA Interim/GDAS data and the PROD3 model, so far used for simulations. The thick error bars represent the standard deviation and the thin error bars the peakto-peak values of relative difference at each altitude bin. The dotted vertical line marks the $2000 \mathrm{~m}$ line, at which the observatories are placed.

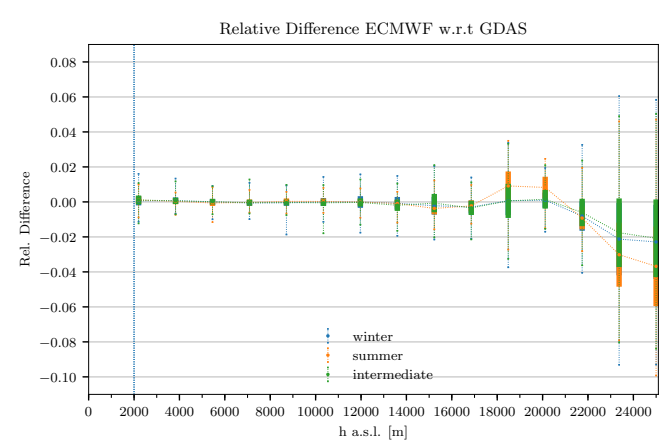

(a) North

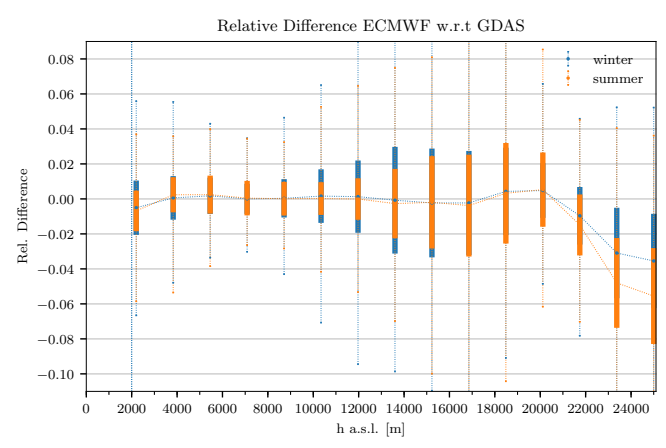

(b) South

Figure 4: Relative difference between the computed density from ECMWF-ERA Interim and GDAS data. The thick error bars represent the standard deviation and the thin error bars the peak-to-peak values of relative difference at each altitude bin. The dotted vertical line marks the $2000 \mathrm{~m}$ line, at which the observatories are placed. 